\title{
Blood Blister-like Aneurysm Treated with Coil Embolization without Stent Assistance
}

\author{
Seongwoo Lee, Kyung-Jae Park, Shin-Hyuk Kang, Dong-Hyuk Park \\ Department of Neurosurgery, Korea University Medical Center, Anam Hospital, Korea University College of Medicine, Seoul, Korea
}

Received: March 10, 2019

Accepted: March 26, 2019

\section{Corresponding Author:}

Dong-Hyuk Park, M.D., Ph.D.

Department of Neurosurgery, Korea University Medical Center, Anam Hospital, Korea University College of Medicine, 73 Inchonro Sungbuk-gu, Seoul 02841, Korea

Tel: +82-2-920-5729

Fax: +82-2-929-0629

E-mail: doctorns@korea.com

\begin{abstract}
We report a case of blood blister-like aneurysm treated with coil embolization without stent assistance. A 34-year-old man with a medical history of hypertension presented with subarachnoid hemorrhage. Initial digital subtraction and computed tomography angiography did not demonstrate definite vascular pathologies. Two weeks later, follow-up angiography showed a wide neck aneurysm on the dorsal wall of left distal internal carotid artery, which was not found on the first angiograms. This blood blister-like aneurysm was successfully treated with coil embolization without stent assistance, and the regrowth or recanalization has not been identified for 6 months. While both surgical and endovascular procedures are quite challenging, endovascular coiling with stent assistance for this aneurysm is generally recommended. But endovascular coiling without stent assistance may be attempted for instances such as relatively narrow neck and young age.
\end{abstract}

Keywords: Blood blister-like aneurysm; Internal carotid artery; Subarachnoid hemorrhage; Endovascular treatment; Saccular embolization

\section{INTRODUCTION}

"Blood Blister-like aneurysm" arises on the dorsal wall of internal carotid artery (ICA), and uniquely occurs at non-branching sites $^{1,3}$. It has thin and fragile wall that requires special care to prevent premature rupture ${ }^{1,3)}$. The characteristic of weak wall and broad neck makes both surgical and endovascular procedures quite challenging ${ }^{3}$. For an endovascular procedure, stent-assisted coil embolization is usually performed, because blood blister-like aneurysms usually have a wide neck, which increases the risk of coil herniation into the parent artery ${ }^{5)}$. The stent before endovascular coil deployment prevents unwanted movements of the microcatheter ${ }^{4)}$. But, in some cases, endovascular coiling may be performed without stent or balloon assis- tance $^{6}$. Here, we present a case of blood blister-like aneurysm treated with endovascular coiling without stent assistance.

\section{CASE REPORT}

A 34-year-old man with hypertension medical history came to the emergency room of our hospital with severe headache in left temporal region. On arrival, the patient displayed an alert mentality on neurologic examination. Computed tomography (CT) showed diffuse subarachnoid hemorrhage (SAH) on basal cistern and bilateral sylvian fissure (Fig. 1). However, on initial cerebral angiography, there was no abnormal finding related with SAH (Fig. 2 ). We decided on a strategy of conservative management in the intensive care unit and follow-up angiography several days later.

Copyright (C) 2019 The Korean Neurointensive Care Society

This is an Open Access article distributed under the terms of the Creative Commons Attribution Non-Commercial License (http://creativecommons.org/licenses/by-nc/4.0/) which permits unrestricted non-commercial use, distribution, and reproduction in any medium, provided the original work is properly cited. 
Two days later, the patient underwent CT angiography, which showed no demonstrable aneurysm. On the hospitalization day 4, the patient was transferred to the general ward from intensive care unit and conservative management was maintained. After transfer to the general ward, the patient's headache gradually subsided, but on hospitalization day 13, relapse of the headache occurred. We performed a second conventional angiography on hospitalization day 15 . The follow-up angiography revealed a ruptured aneurysm with a wide neck at the supraclinoid segment of left internal carotid artery (ICA) (Fig. 3). The aneurysm was on a non-branching site

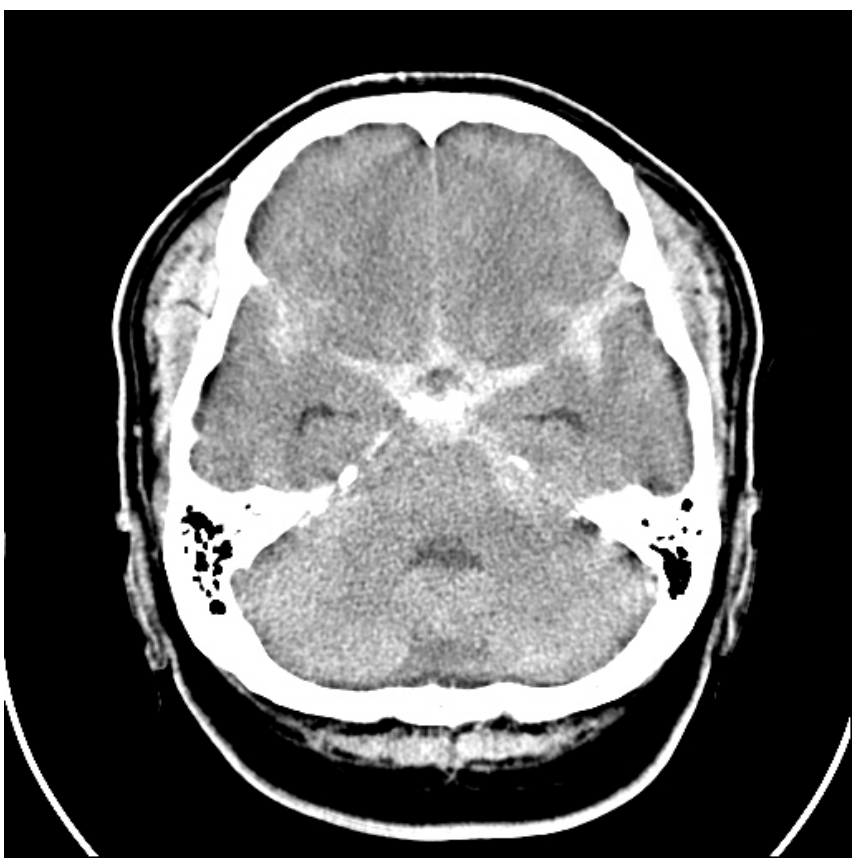

Fig. 1. Brain CT scans showing a diffuse subarachnoid hemorrhage on the basal cistern and bilateral sylvian fissure. and measured $4.3 \times 4.3 \times 3.2 \mathrm{~mm}$ wide in the antero-superior direction. The aneurysm was considered to be a blood blister-like aneurysm due to its location of dorsal wall of ICA and non-branching site, and rapid progression in size in just fifteen days.

We performed endovascular coiling without stent assistance (Fig. 4). Generally, stent assistance is recommended for the treatment of blood blister-like aneurysms with endovascular coiling. In this case, the patient was young and a repeat angiography showed a relatively narrow aneurysm neck to prevent the coils from herniation into the parent artery. After placement of a 6 French introduc-

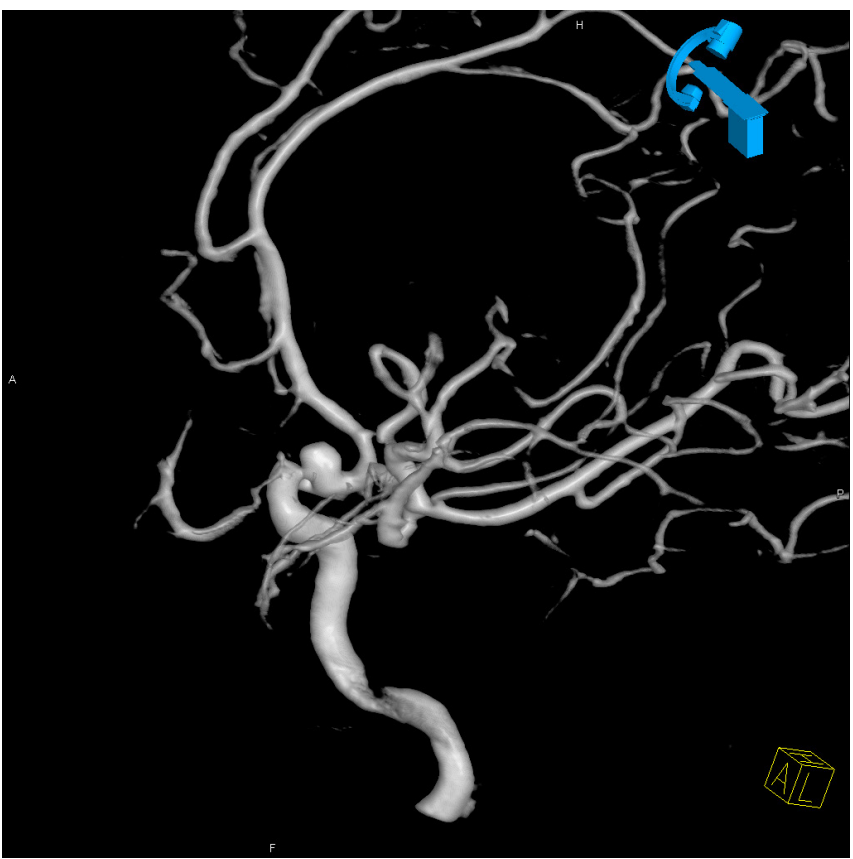

Fig. 3. Fifteen days later, second angiography revealed a ruptured aneurysm with a wide neck at the dorsal wall of supraclinoid segment of left internal carotid artery, the so-called blood blister-like aneurysm.

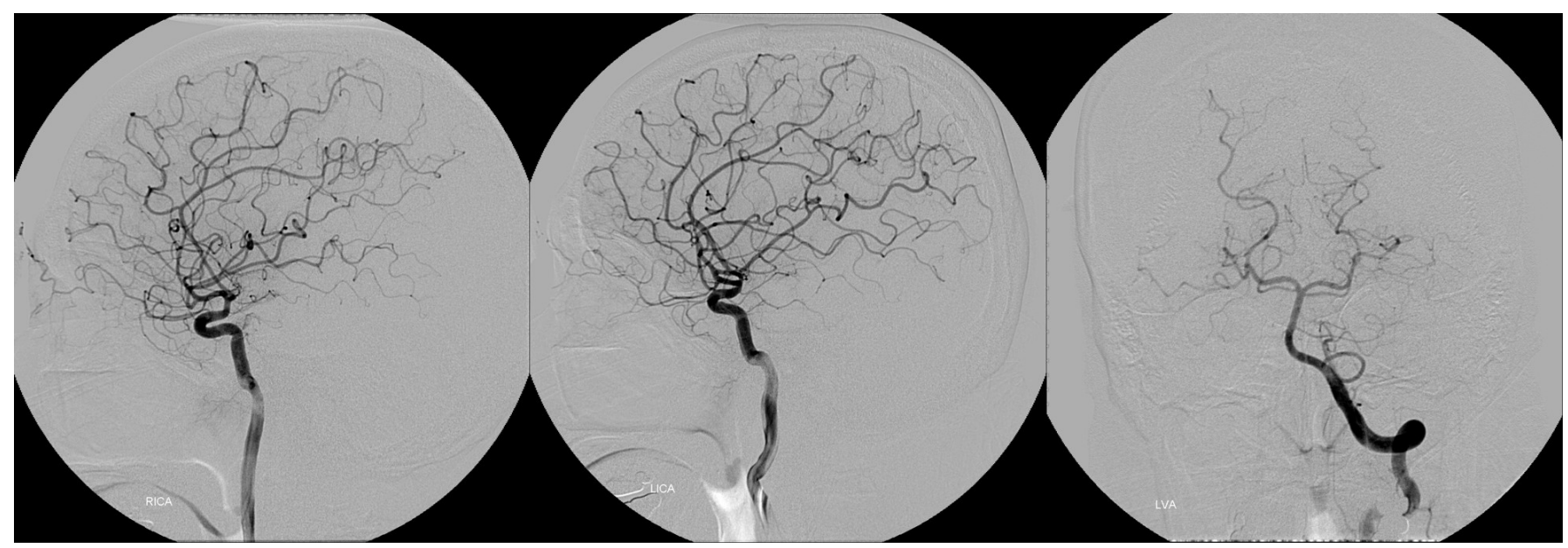

Fig. 2. Initial conventional angiography did not demonstrate definite vascular abnormality. 


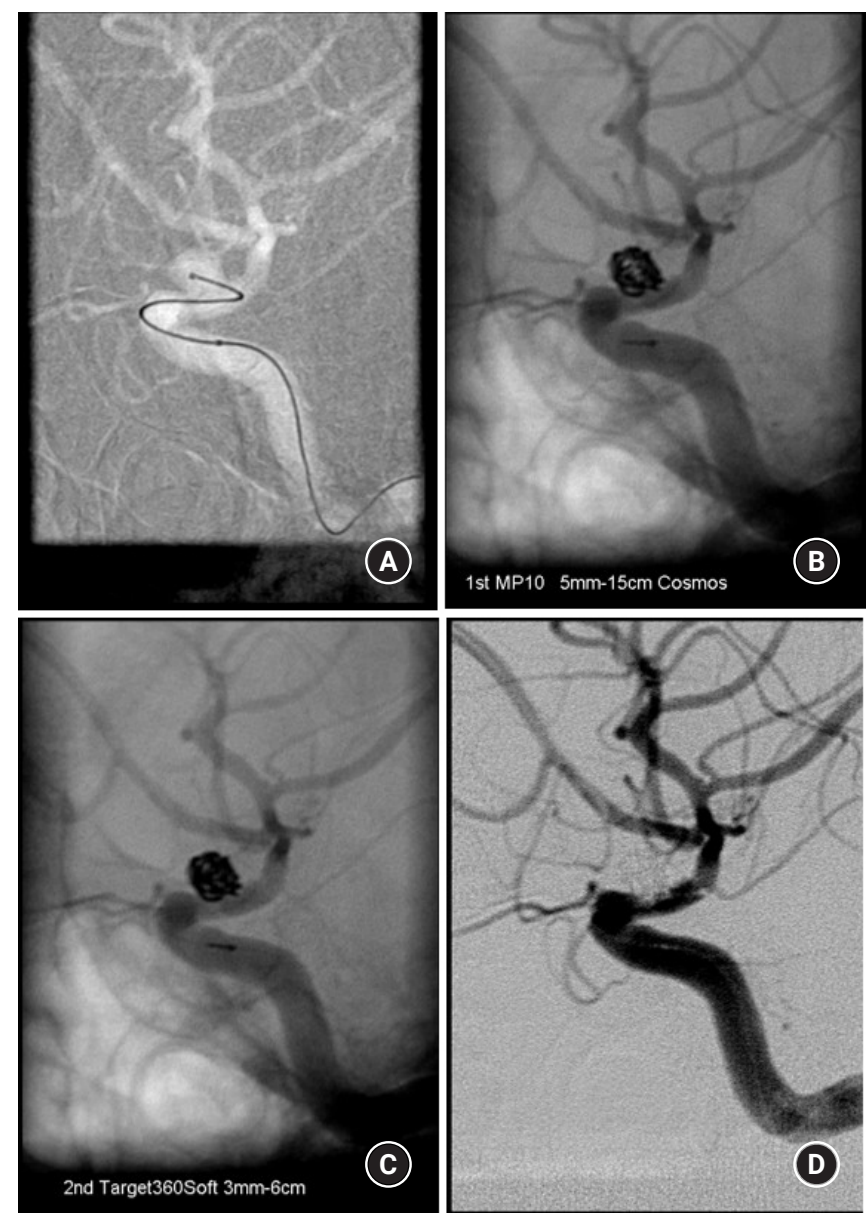

Fig. 4. The patient underwent an endovascular coiling without stent assistance. (A)After a microcatheter was positioned in blister aneurysm, (B)the first Guglielmi detachable coil was deployed. (C) Then, a second coil was followed. (D)Post-procedural angiography showed near complete coil embolization without compromising the parent artery.

ing sheath on the right femoral artery, a 6 French guiding catheter (Envoy; Cordis Endovascular Systems, Miami Lakes, FL, USA) was placed at the distal cervical segment of the left ICA. First, careful deployment of a Guglielmi Detachable Coil (GDC) (microplex $10,5 \times 15 \mathrm{~cm}$ cosmos complex) was attempted in the aneurysm sac. A second GDC (target 360 soft $3 \times 6 \mathrm{~cm}$ ) was followed. These coils were placed successfully within the aneurysm sac without compromising the parent artery. The post-procedural angiography showed near complete coil embolization, and no evidence of thrombo-embolic complications.

The post-coiling course was uneventful. The patient was discharged on day 20 the after procedure without neurological deficit. We performed repeated angiography 6 months after endovascular coiling, and the regrowth of coiled aneurysm has not been identified yet.

\section{DISCUSSION}

Aneurysms located at the non-branching site on the dorsal wall of the supraclinoid segment of ICA were first reported in 1969 by Sundt et $\mathrm{al}^{5}$. They are rare, comprising $0.9 \% \sim 6.5 \%$ of all intracranial aneurysms $\mathrm{s}^{5}$. Various surgical and endovascular treatments have been suggested for the treatments of blood blister-like aneurysms, including direct clipping, wrapping, clipping with wrapping material, clipping with Sundt encircling graft clips, proximal occlusion of the ICA, and proximal endovascular occlusion of the ICA followed by surgical clipping of the ICA distal to the aneurysm ${ }^{2,45}$. But, despite of these diverse endeavors to treat, blister aneurysm is quite challenging for both surgical and endovascular treatments ${ }^{3)}$.

Few pathologic examinations have been performed for blood blister-like aneurysms, and it has been reported as focal wall defects covered by a thin layer of fibrous tissue and adventitia and lack of usual collagenous layer ${ }^{2,5}$. This unique nature of aneurysm wall leads to a marked weakness and high risk of premature rupture during surgical and endovascular procedures ${ }^{1,3)}$.

Although the lesion of this case displayed a relatively larger size than those described in other reports, it is considered as a blood blister-like aneurysm, because the location of aneurysm in our case corresponds to the unique feature of blister-like aneurysms. Furthermore, the size and morphology of the aneurysm changed rapidly in just 15 days. Blood blister-like aneurysm may show rapid progression in size and morphology in repeated angiography ${ }^{2,4)}$. In our case, the initial conventional digital subtraction angiography and $\mathrm{CT}$ angiography did not show a definite vascular pathology including aneurysm. But on repeated angiography performed on hospitalization day 15 , a definite sizable dorsal wall aneurysm was identified on the ICA. The rapid growth in size and morphology in short intervals was probably related with the weak wall of the blister-like aneurysm and hemodynamic stress coming from their location at non-branching site and dorsal portion of supraclinoid $\mathrm{ICA}^{3)}$.

This marked weakness of aneurysm wall is associated with the difference of treatment and prognosis from saccular aneurysms. Besides, the location of blister-like aneurysm and the nature of adhesion to brain tissue lead to high risk of premature rupture ${ }^{1)}$. Standard surgical exploration and micro-dissection around the aneurysm may be more dangerous than saccular aneurysm. The difficulty of surgical procedure results in high mortality of blood blister-like aneurysms. The mortality of SAH with ruptured blister-like aneurysm is $57 \%$, and overall mortality of ruptured and unruptured blister-like aneurysm is $20 \%^{1)}$.

Endovascular treatment has been studied for an alternative 
method for obliteration of blood blister-like aneurysms. The unique feature of broad neck and weak wall of blister-like aneurysms makes endovascular procedures technically challenging, too. Stent assistance is usually recommended because of the broad neck of blood blister-like aneurysms. Without stent assistance, not only the probability of coil herniation into the parent artery may increase ${ }^{5)}$, but also the deployment of detachable coil in blister aneurysm itself can be very difficult technically. In our case, we considered to perform endovascular coiling with stent assistance. But, on the angiography, somewhat narrow portion of aneurysm neck was identified and anticipated to support the packed coils from herniation into the parent artery. Moreover, the relatively large size of blister-like aneurysm was favorable for holding the packed coils in the aneurysm. In general, it is usually accepted that the positioning of stent before endovascular coiling is safe to avoid unexpected movements of the microcatheter. But, we could not rule out the possibility of failure to super-select the aneurysm with GDC due to the pre-positioned stent and the location of blister-like aneurysm. Furthermore pre- and post-coiling anti-platelet agents are necessary for intracranial stent due to high tendency of stimulating platelet aggregation, and this disadvantage leads to risk of blister-like aneurysm rebleeding. The "jailing technique" may be considered to perform in the unstable condition such as blister-like aneurysms. In the jailing technique, a stent is deployed across the aneurysm neck after the first microcatheter has been placed in the aneurysm. This procedure effectively "jails" the first microcatheter between the stent and the arterial wall so that access to the aneurysm is not lost after stent placement. The jailing technique has an advantage in comparison with formal stent-assisted coiling, that the access to the aneurysm is not lost after stent placement. But jailing technique also requires long-term use of anti-platelet agents.

Recurrence or regrowth is quite common after endovascular coiling for blister-like aneurysms ${ }^{3}$. Repeated post-coiling evaluation including conventional angiography is necessary, and our patient is scheduled to regular follow-up conventional angiography.

\section{CONCLUSION}

SAH with normal conventional angiography needs to be considered the possibility of blood blister-like aneurysm, and careful examination with repeated angiography is required to prevent misdiagnosis. Various surgical and endovascular treatments have been documented for blister-like aneurysms. Stent assistance is generally recommended for the treatment of blister-like aneurysms with coil embolization. But, endovascular coiling without stent assistance may be attempted for some instances such as relatively narrow neck and young age.

\section{CONFLICT OF INTEREST}

No potential conflict of interest relevant to this article was reported.

\section{REFERENCES}

1. Ahn JY, Cho JH, Jung JY, Lee BH, Yoon PH. Blister-like aneurysms of the supraclinoid internal carotid artery. challenging endovascular treatment with stent-assisted coiling. J Clin Neurosci 2008; 15:1058-1061

2. Ahn JY, Kwon SO, Joo JY. Dorsal internal carotid artery aneurysm treated by coil embolization--case report. Neurol Med Chir (Tokyo) 2001;41:603-605; discussion 6.

3. Andaluz N, Zuccarello M. Blister-like aneurysms of the anterior communicating artery. a retrospective review of diagnosis and treatment in five patients. Neurosurgery 2008;62:807811; discussion 11.

4. Jha AN, Gupta V. Blister aneurysms. Neurol India 2009;57:23.

5. Korja M, Rautio R, Valtonen S, Haapanen A. Primary treatment of ruptured blood blister-like aneurysms with stent-assisted coil embolization: report of two cases. Acta Radiol 2008;49:180-183.

6. Matsubara N, Miyachi S, Tsukamoto N, Izumi T, Naito T, Haraguchi K, et al. Endovascular coil embolization for saccular-shaped blood blister-like aneurysms of the internal carotid artery. Acta Neurochir (Wien) 2011;153:287-294. 